\title{
Color Measurements with a Consumer Digital Camera Using Spectral Estimation Techniques
}

\author{
Martin Solli ${ }^{1}$, Mattias Andersson $^{2}$, Reiner Lenz ${ }^{1}$, and Björn Kruse ${ }^{1}$ \\ ${ }^{1}$ Center for Creative Media Technology, ITN, Campus Norrköping, Linköpings Universitet, \\ SE-601 74 Norrköping, Sweden \\ martin.solli@medietekniker.se, \{reile, bjokr\}@itn.liu.se \\ http://www.media.itn.liu.se \\ ${ }^{2}$ Digital Printing Center, Mid Sweden University, \\ SE-891 18 Örnsköldsvik, Sweden \\ mattias.andersson@miun. se \\ http: / / www.miun.se/dpc
}

\begin{abstract}
The use of spectrophotometers for color measurements on printed substrates is widely spread among paper producers as well as within the printing industry. Spectrophotometer measurements are precise, but timeconsuming procedures and faster methods are desirable. The rapid development of digital cameras has opened the possibility to use consumer digital cameras as substitutes for spectrophotometers for certain applications such as production control. Two methods for estimating the reflectance properties of objects from camera RGB measurements using linear estimation techniques combined with linear and non-linear constraints are presented. In the experiments, we have investigated if these techniques can be used to measure the reflectance properties of flat objects such as printed pages of paper. Reflectances were converted to CIELAB color values, and the minimization of color errors were evaluated with CIE color difference formulas. Our experiments show that a consumer digital camera can be used as a fast and inexpensive alternative to spectrophotometers for color measurements on printed substrates.
\end{abstract}

\section{Introduction}

The standard instrument to measure the reflectance properties of an object is a spectrophotometer. This gives very precise results but the measurements are done point-wise. The procedure is therefore time-consuming and it is difficult to measure spatial variations or to obtain image information. Furthermore, it is a comparatively expensive instrument. The rapid improvement of digital cameras (and their rapidly falling prices) on the other hand has triggered considerable interest in the question if these cameras can be used as measurement devices to obtain sufficiently accurate color information. If this is possible, then one could produce fast measurements for a large number of measurement points in parallel at a relatively low cost. In this paper, we describe some of our experiments in which we investigated the properties of a standard consumer digital camera as a colorimetric measurement device.

We describe two methods to estimate reflectance spectra from RGB measurements. The first method, called Camera Estimation, first estimates the response properties of 
the camera. This camera model is then used to estimate the reflectance spectra of object points. Known techniques will be evaluated, and a variation called MultiSensor will be introduced and investigated. The second method, here named Direct Spectral Estimation, estimates the reflectance spectra directly from the RGB vectors. Since the CIE color difference formulas are widely used, CIELAB values were calculated from the estimated spectra in order to simplify the evaluation and calculate the color difference between reference and reproduced colors.

\section{Background}

One way to use a camera as a colorimeter is to find a model, often some type of regression, which directly maps RGB values to CIELAB values. Examples are described in Hong et. al [1], Wu et. al. [2], MacDonald \& Ji [3], Brydges et. al. [4], and Orava et. al. [5]. For printed substrates, this approach has been shown to produce the best results when calibration and measurements are made on samples printed with the same combination of printer and substrate (see for example Hägglund [6]). Moreover, the dependence of the CIELAB values on the selected white-point is another disadvantage which implies that the regression has to be recomputed for new selections of the white-point.

Instead of estimating CIELAB values from camera RGB values one can try to estimate the spectral distribution. Then it is possible to compute the coordinate of the color in different color spaces, such as CIELAB. The availability of the spectral distribution also allows different types of post-processing, for instance simulating the color coordinates under different illuminations. One way to compute such an spectral estimation is to approximate the sensitivity functions of the RGB filters in the camera and use this to estimate the spectral distributions. A tunable monochromatic light source can generate narrow-band light distributions of different wavelengths which can be measured by the camera sensor. The recorded response vectors characterize the transfer functions of the camera filters. Examples of this approach can be found in $\mathrm{Wu}$ et. al. [2] and MacDonald \& Ji [3]. Here, we will use another common approach where targets containing several colored patches will be acquired with the camera. The recorded camera RGB values will be combined with color values measured by a spectrophotometer to find the spectral characteristics of the camera. This approach avoids the usage of the monochromator and in addition, broadband techniques are often less sensitive to noise than corresponding narrowband approaches.

One common method for finding color filter characteristics is to use a method called Projections Onto Convex Sets (POCS), see for example Sharma [7]. POCS is an estimation technique that combines a priori information with the measurements to obtain the estimates. The disadvantage of this method is that it can be strongly dependent on a priori knowledge. Without a good estimation, the result can be poor.

Finally, we mention methods using Neural Networks (see Ramanath et. al. [8] as a recent example) that use non-linear techniques to "learn" the desired relations from examples. Neural Networks require a significant amount of time for learning the structure of the data, but once trained, the execution time of the network is comparable to other methods. 


\section{Camera as a Colorimeter}

In this section, we describe two methods that are used to estimate the reflectance spectra from the RGB measurements. In the following discussion, we will use two matrices $\mathbf{R}$ and $\mathbf{C}$ : in the matrix $\mathbf{R}$ we collect the vectors with the spectral reflectance for each color patch as measured with a spectrophotometer. The matrix $\mathbf{C}$ contains the vectors with the corresponding RGB values from the camera. The goal is to find a transformation that maps $\mathbf{C}$ to $\mathbf{R}$ and we will describe two approaches. The first method follows the traditional approach which is to invert the camera and estimate the transfer functions. We will mainly follow previous approaches, but also extend with a multi-sensor-type generalization.

The second method can be seen as the inverse of the first approach. Color spectra will be reproduced, but in this case, we treat it as a one-step estimation problem.

\subsection{Camera Estimation}

The first method requires that the cameras sensitivity functions are estimated first. We describe the camera as a linear system with a matrix $\mathbf{W}$, containing three rows, one for each filter function. This gives the following model:

$$
\mathbf{C}=\mathbf{R W}
$$

The matrices $\mathbf{C}$ (RGB values) and $\mathbf{R}$ (spectral reflectance functions) are known and Eq. (1) has to be solved for the matrix W. We choose the Moore-Penrose pseudo inverse, $\operatorname{pinv}(\mathbf{R})$ of $\mathbf{R}$, to obtain the following estimation of $\mathbf{W}$ :

$$
\mathbf{W}=\operatorname{pinv}\left(\mathbf{R}^{\prime}\right)^{\prime} \mathbf{C}
$$

Since $\operatorname{pinv}(\mathbf{R})=\left(\mathbf{R}^{\prime} \mathbf{R}\right)^{-1} \mathbf{R}^{\prime}$ we see that: $\mathbf{R W}=\mathbf{R} \operatorname{pinv}\left(\mathbf{R}^{\prime}\right)^{\prime} \mathbf{C}=\mathbf{R}\left(\left(\mathbf{R R}^{\prime}\right)^{-1} \mathbf{R}\right)^{\prime} \mathbf{C}=$ $\mathbf{R}^{\prime}\left(\mathbf{R R}^{\prime}\right)^{-1} \mathbf{C}=\mathbf{C}$ and in the absence of noise, this is an exact solution. However, under real-world conditions, the result can be difficult to use. This noise sensitivity can be incorporated into the estimation, for example using the Singular Value Decomposition (SVD), Principal Component Analysis (PCA) or Wiener estimation techniques (see Hardeberg [9] for one description of the PCA-technique).

Further improvements in the spectral sensitivity estimation can be gained by introducing additional constraints. Three constraints proposed by Finlayson et. al. [10], later adopted and slightly modified by Barnard and Funt [11] were used in our estimation. The first constraint is related to the Fourier transform. Here a smoothness constraint is introduced by restricting the spectral sensitivity function to linear combinations of a few low-degree Fourier basis functions. Since a device cannot have a negative response to a stimulus, the second constraint is the non-negativity of the functions involved. The third constraint states that the number of peaks in a sensor curve is small. This is referred to as the modality constraint. An alternative to the incorporation of these constraints is a method called Parametric Fitting, proposed by Thomson \& Westland in [12]. This method results in similar robust estimates, but the constraints are through the adoption of a low-dimensional parametric model.

In the next step, the estimated camera characteristics are used to estimate the input spectra from measured camera values. The simplest way is to use a pseudo-inverse approach but this turns out to be very noise sensitive. One solution (see Hardeberg 
[9]) is to take advantage of a priori knowledge about the spectral reflectance functions that are to be constructed. A set of smooth basis functions were constructed. Starting with a large number of different colors, we selected those colors having the most different spectral distributions. This selection procedure is described in Section 4. Singular Value Decomposition is then carried out with these colors to extract only the most significant values. Finally, Fourier based smoothness criteria and positivity constraints are applied to further improve the result.

Inspired by multispectral digital cameras with more than three filters, we found that by using combinations of existing RGB filters, the estimation of spectra from camera values could be improved. The matrix $\mathbf{C}$ introduced in Equation 1 has the form:

$$
\mathbf{C}=\left[\begin{array}{lll}
R & G & B
\end{array}\right]
$$

We extend $\mathbf{C}$ by adding the sums of color channel pairs, and obtain a new matrix C:

$$
\mathbf{C}=\left[\begin{array}{lllll}
\mathrm{R} & \mathrm{G} & \mathrm{B} & \mathrm{R}+\mathrm{G} & \mathrm{R}+\mathrm{B} \quad \mathrm{G}+\mathrm{B}
\end{array}\right]
$$

This variation decreased the maximum error obtained in the evaluation tests.

\subsection{Direct Spectral Estimation}

In the second approach, we ignore the role of the camera filters and directly consider the desired relation between the $\mathbf{C}$ and $\mathbf{R}$, matrices. The model we use can be described as follows: Given the matrices $\mathbf{C}$ and $\mathbf{R}$ find the best matrix $\mathbf{M}$ with

$$
\mathbf{R}=\mathbf{C M}
$$

Again, $\mathbf{R}$ contains spectral reflectance functions, $\mathbf{C}$ contains the corresponding RGB values, and $\mathbf{M}$ is a linear operator. Using the pseudo-inverse, $\mathbf{M}$ can be expressed as:

$$
\mathbf{M}=\mathbf{R}^{\prime} \operatorname{pinv}\left(\mathbf{C}^{\prime}\right)
$$

where $\operatorname{pinv}\left(\mathbf{C}^{\prime}\right)$ is the Pseudo-Inverse of $\mathbf{C}^{\prime}$. In this method, the illumination is removed from the spectral distribution before the calculations are performed. Once again, a constraint on the Fourier transform can be used to improve the result by smoothening the functions. Here we also introduce an additional constraint. Since the illumination is removed from the spectral distribution, all spectral values in the result must be within the interval from zero to one.

Once the matrix $\mathbf{M}$ is known, the spectral distributions are calculated by:

$$
\mathbf{R}=\mathbf{C M}
$$

Again, we can improve the result with a constraint on the Fourier transform or by forcing the reconstructed spectra to be linear combinations of a spectral basis. We constructed such a basis by first choosing those color spectra that had different spectral distributions. Thereafter Singular Value Decomposition was used to extract the most significant basis vectors. 


\section{Color Patches}

Eight pages from NCS Color Atlas 96 [13], selected according to Fig. 1, were used. This resulted in 365 color patches in total. All these colors were used for evaluation. But for characterization, either a small number of these NCS patches were used, or charts printed with inkjet on matte-coated substrate.

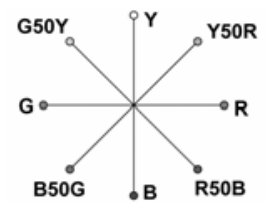

Fig. 1. The selection of NCS pages used in this study

We followed the proposal by Hardeberg [9] to select the optimal colors to be used in spectral sensitivity characterization. The strategy for the selection of the reflectance samples $\mathrm{r}_{\mathrm{S} 1}, \mathrm{r}_{\mathrm{S} 2}, \mathrm{r}_{\mathrm{S} 3}, \ldots$ which are most significant in the characterization of the camera is as follows: Starting from the full set of all available spectral reflectance functions $r_{p}, p=1 \ldots$, we first select that $r_{S 1}$ which has maximum RMS value:

$$
\left\|r_{S 1}\right\| \geq\left\|r_{P}\right\| \text { for } \mathrm{p}=1 \ldots \mathrm{P}
$$

Next, we select $r_{S 2}$ which minimizes the condition number of $\left[r_{S 1} r_{S 2}\right]$, the ratio of the largest to the smallest singular value. Denoting $w_{\min }(\mathrm{X})$ and $w_{\max }(\mathrm{X})$ as the minimum and maximum singular values of a matrix $\mathrm{X}$, this minimization may be expressed by the following expression:

$$
\frac{w_{\text {max }}\left(\left[r_{S 1} r_{S 2}\right]\right)}{w_{\text {min }}\left(\left[r_{S 1} r_{S 2}\right]\right)} \leq \frac{w_{\text {max }}\left(\left[r_{S 1} r_{P}\right]\right)}{w_{\text {min }}\left(\left[r_{S 1} r_{P}\right]\right)} \text { for } \mathrm{p}=1 \ldots \mathrm{P}, \mathrm{p} \neq \mathrm{s} 1
$$

Further sample spectra are added according to the same rule:

$$
\frac{w_{\max }\left(\left[r_{S 1} r_{S 2} \ldots r_{S i}\right]\right)}{w_{\text {min }}\left(\left[r_{S 1} r_{S 2} \ldots r_{S i}\right]\right)} \leq \frac{w_{\max }\left(\left[r_{S 1} r_{S 2} \ldots r_{S i-1} r_{P}\right]\right)}{w_{\min }\left(\left[r_{S 1} r_{S 2} \ldots r_{S i-1} r_{P}\right]\right)} \text { for } \mathrm{p}=1 \ldots \mathrm{P}, \mathrm{p} \notin\{\mathrm{s} 1, \mathrm{~S} 2, \ldots \mathrm{Si}-1\}
$$

For each iteration step, a reflectance spectrum that is as different as possible from the already selected spectra is chosen.

\section{Equipment and Measurements}

In our experiments we used a Canon EOS 10D digital camera with a Canon EF 50mm 1:1.8 II lens. The image sensor is a CMOS sensor, with a Red, Green or Blue filter on top of each photodiode. Each captured image is saved in the Canon RAW image format. In an initial test, we measured the response of the camera to inputs of varying intensity. For this purpose, a set of pictures with varying portions of black and white 
pixels were displayed on a laptop monitor, and photos were captured and saved in both RAW and JPG image format. In Fig. 2, mean intensity values from each type of filter are plotted against the laptop monitor coverage.

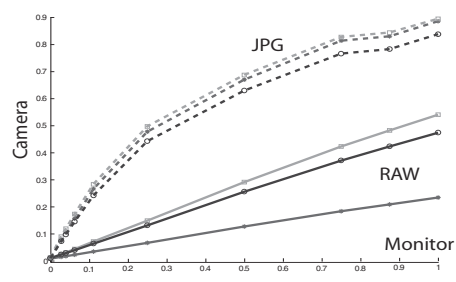

Fig. 2. Mean intensity values from each color channel (R, G and B) and image format (RAW and JPG) plotted against laptop monitor coverage

The result shows that the JPG responses are not linear to the input optical energy. This is the result of the internal processing of the raw images with the goal to produce "more appealing" pictures. Two common processing steps are white balancing and gamma curve compensations (see Orava et. al. [5] for a discussion of these properties). The RAW image on the other hand, displays output intensities that are almost perfectly linear to the input optical energy. This indicates that using the RAW format is an advantage if the camera is to be used as a measuring instrument.

The illumination was provided by the Minispectra light cabinet from Largo AB. It provides a selection of different light sources but here we only used the Daylight (D65) and light bulb (A). The spectral distributions for these sources are shown in Fig. 3. Notice that the illumination D65 has a different spectral distribution compared to the one defined by CIE. Experiments showed that the sharp peaks in the illumination from Largo could cause problems in the spectral estimation process. This observation is confirmed by many authors, e.g. Romero et. al. [14] and Hardeberg et. al. [15], who have reported that the estimation becomes more difficult if fluorescent illumination is used, especially if it has sharp peaks in the spectral radiance.

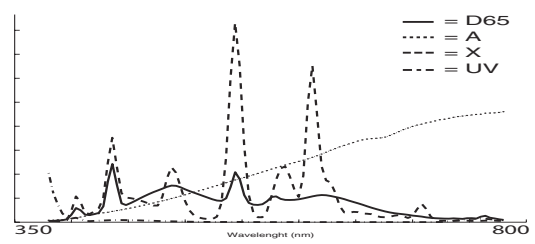

Fig. 3. The spectral distributions of the different light sources available in the light cabinet

By using a measurement geometry were the illumination angle is set to $10^{\circ}$, and the measurement angle to $55^{\circ}$, problems with non-uniform illumination and gloss effects were avoided as much as possible. Before images were used in calculations, they were compensated for optical and natural vignetting. The spectrophotometer measurements where carried out with a PR-650 SpectraColorimeter for inkjet charts, and a MacBeth ColorEye 700 for NCS charts. The illumination was measured with a 
reflectance standard (SRS-3 from Photo Research) and obtained values were used as white reference in the conversion between CIEXYZ and CIELAB values.

\section{Results}

In the Camera Estimation (CE) method, 22 inkjet colors with varying spectral distributions were used for calibration. The evaluation is based on 365 NCS colors, and illumination A was used for both calibration and evaluation. In the Direct Spectral Estimation (DSE) method, 25 NCS colors with varying spectral distributions were used for calibration, but both illumination A and D65 were used. Hence, 50 calibration colors were used. The evaluations were carried out using 365 NCS colors under illumination A. Fig. 4 shows the resulting filter functions.

\begin{tabular}{|l|c|c|}
\hline CE (RGB-sensor) & CE (MultiSensor) & DSE \\
\hline
\end{tabular}

Fig. 4. Filter functions for original RGB-sensor, MultiSensor, and Direct Spectral Estimation

These functions are then used to reproduce the input spectra from measured camera values. Fig. 5 shows two examples of reproduced spectra.

\begin{tabular}{|c|c|c|c|}
\hline & CE (RGB-sensor) & CE (MultiSensor) & DSE \\
\hline $\begin{array}{c}\text { NCS } \\
\text { S 1040-R50B }\end{array}$ & $\begin{array}{lll}400 & 500 \quad 600 \quad 700\end{array}$ & ${ }_{400500600700}^{0.5}$ & 100500600700 \\
\hline $\begin{array}{c}\text { NCS } \\
\text { S 1050-G50Y }\end{array}$ & & 400500600700 & $\begin{array}{llll}0.5 & \\
400500600700\end{array}$ \\
\hline
\end{tabular}

Fig. 5. The solid lines represent the original spectra measured with the spectrophotometer and the dashed lines represent the reproduced spectra

In order to simplify the evaluation, CIE Color Matching Functions were used to convert each spectrum to CIEXYZ values. Then CIEXYZ values were converted to CIELAB values and $\triangle \mathrm{E}$, the difference in CIELAB color space, was calculated between measured and reproduced colors. The result can be seen in Table 1 .

Table 1. Mean and maximum color difference for the three methods

\begin{tabular}{|l|l|c|c|c|}
\hline & & CE (RGB-sensor) & CE (MultiSensor) & DSE \\
\hline$\Delta \mathbf{E}$ & mean & 2.84 & 2.84 & 3.50 \\
\hline & $\max$ & 12.30 & 9.81 & 9.14 \\
\hline
\end{tabular}


In Fig. 6, the reproduction errors for both the MultiSensor method and the Direct Spectral Estimation method are separated into different $\Delta \mathrm{E}$ intervals.

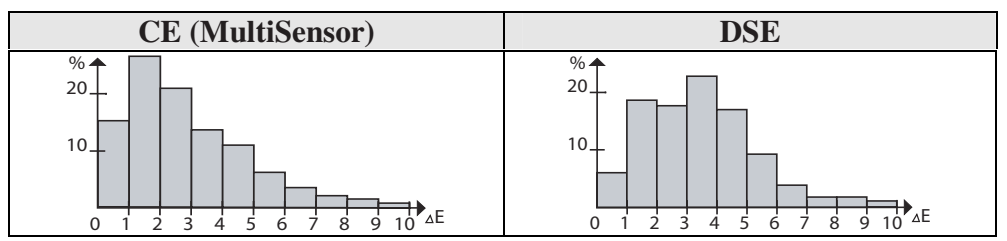

Fig. 6. $\Delta \mathrm{E}$ distributions for MultiSensor and Direct Spectral Estimation

For the MultiSensor method, $61.9 \%$ of the reproduced spectra have an error less than $3 \Delta \mathrm{E}$ units. Only $7.7 \%$ have a color error greater than $6 \Delta \mathrm{E}$. When Direct Spectral Estimation is used, $42.2 \%$ of the reproduced spectra have an error less than 3 $\Delta \mathrm{E}$, and only $8.7 \%$ have a color error greater than $6 \Delta \mathrm{E}$. In Fig. 7 , the reproduction errors are separated into different locations in the color space, corresponding to different pages in the NCS color system.

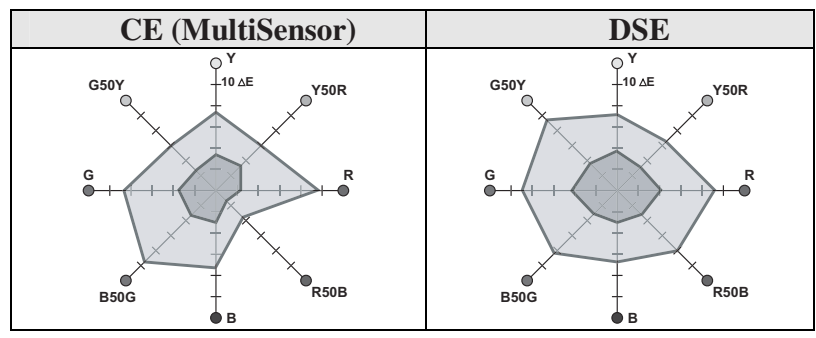

Fig. 7. Color difference separated into different NCS pages. Inner circle represents mean values, the outer maximum values

For the MultiSensor method, the largest error can be found on the red page, but at the same time the neighboring page, the red and blue, has the smallest error. The conclusion is that the reproduction error varies considerably between different locations in color space. When Direct Spectral Estimation is used, the color differences for both mean and maximum, are almost the same for all pages.

Preliminary experiments with the D65 equivalent illumination shown in Fig. 3 gave the following results: Mean and maximum color difference with Camera Estimation (MultiSensor) was 10.05 and $18.96 \Delta \mathrm{E}$ units. With Direct Spectral Estimation the mean and maximum error was calculated as 4.81 and $13.01 \Delta \mathrm{E}$.

The results from the color measurement methods presented in this article can be compared to results presented by other authors. Hardeberg [9] calculated a mean $\Delta \mathrm{E}$ value about 6 , and a maximum value in the region of $16 \Delta \mathrm{E}$. Orava et. al. [5] present a mean error greater than $10 \Delta \mathrm{E}$, but they also mention that with a high-quality camera, the color difference can be as low as $5 \Delta \mathrm{E}$ units. Wu and Allebach [2] achieved a mean color error in the region of $2 \Delta \mathrm{E}$. All these results were obtained with regression-based methods. Instead, if a spectral estimation technique is used, 
Hardeberg's [9] calculated mean and maximum values were almost 7 respectively 17 $\Delta \mathrm{E}$ units. In the special context of art reproduction Imai et. al. [16] [17] reported mean and maximum $\Delta \mathrm{E}$ errors for a professional three channel camera about 2 and $6 \Delta \mathrm{E}$ units. They used six channels (filters) in their acquisitions.

\section{Conclusions}

We have investigated how a moderately priced digital camera can be used for color measurements. Two different methods to map the camera RGB values to reflectance spectra were presented. We found that the camera used in this work had a linear sensor response to incoming intensity when the RAW picture format was used. We also found that illumination sources with sharp peaks in the spectral distribution, such as fluorescent lamps should be avoided.

The two methods for the estimation of spectral distributions described in this paper produced similar results. Since reflectance spectra are estimated it is possible to estimate the appearance of the target under different illuminations, a result that cannot be obtained with regression methods. Another advantage is that relatively few colors are required in the calibration procedure. In this case, we used only 22 and 25 for each method respectively. A larger number of calibration colors will not necessarily improve the result. It is important, though, to notice that the calibration colors should be selected carefully. One way to do this is to select a set of colors with spectral distributions that are as different as possible from each other.

The best result with the Camera Estimation method was a mean color difference of $2.84 \Delta \mathrm{E}$, and a maximum color difference of $9.81 \Delta \mathrm{E}$. It should be noticed that $61.9 \%$ of the measurements had a $\Delta \mathrm{E}$ below 3 , and only $7.7 \%$ of them had a $\Delta \mathrm{E}$ above 6 . With the MultiSensor, the maximum errors were significantly reduced. This method also seems to be rather sensitive to fluorescent lamps with sharp peaks in the illumination spectra. The best result achieved with the Direct Spectral Estimation method had a mean color difference of $3.51 \Delta \mathrm{E}$, and a maximum color difference of $9.15 \Delta \mathrm{E}$. For this method, $42.2 \%$ of the measurements had a reproduction error below $3 \Delta \mathrm{E}$, and $8.7 \%$ of them had an error above $6 \Delta \mathrm{E}$. This is higher than for the Camera Estimation method, but the advantage with this method is that it is less sensitive to peaks in the illumination spectra. With fluorescent lamps, both calibration and evaluation can be made with only a little degeneration compared to a more uniform illumination source using this method.

For both methods the largest measurement errors were obtained for the same type of colors. The best reproduction was found for neutral colors, followed by bright colors. Dark and highly saturated colors were the most difficult to measure.

\section{Acknowledgments}

This article is based on a Master thesis work which was formulated and initiated by MoRe Research in Örnsköldsvik, Sweden and it has been carried out with financial support from the Swedish national research program T2F. 


\section{References}

1. Hong, Guowei, et.al, "A Study of Digital Camera Colorimetric Characterization Based on Polynomial Modeling", Color Research and Applications, Vol. 26, Nr 1, Feb. 2001, (2001).

2. Wu, Wencheng, et. al, "Imaging Colorimetry Using a Digital Camera", The Seventh Color Imaging Conference: Color Science, Systems, and Applications, IS\&T, (1999).

3. MacDonald, Lindsay, \& Ji, Wei, "Colour Characterisation of a High-Resolution Digital Camera", Colour in Graphics, Imaging and Vision (CGIV) Conference, Poitiers, (2002).

4. Brydges, D., et. al, "Application of 3-CCD color camera for colorimetric and densitometric measurements".

5. Orava, Joni, et. al, "Color Errors of Digital Cameras", Color Research and Applications, volume 29, Number 3, June 2004, (2004).

6. Hägglund, Åsa, "Colour gamut and colour sensitivity of a desktop scanner", Diploma thesis, Linköping University, Sweden, (2003).

7. Sharma, Gaurav, "Targetless Scanner Calibration", Journal of Imaging Science and Technology 44: 301-307, (2000).

8. Ramanath, Rajeev, et. al, "Spectral Spaces and Color Spaces", Color Research and Applications, volume 29, Number 1, February 2004, (2004).

9. Hardeberg, Jon Yngve, "Acquisition and Reproduction of Color Images: Colorimetric and Multispectral Approaches", Ecole National Supérieure des Télecommuications, Départment TSI, France, (1999).

10. Finlayson, Graham, et. al, "Recovering Device Sensitivities with Quadratic Programming", The Sixth Color Imaging Conference, Systems, and Applications, IS\&T, (1998).

11. Barnard, Kobus, \& Funt, Brian, "Camera Characterization for Color Research", Color Research and Applications, volume 27, Number 3, June 2002, (2002).

12. Thomson, M., \& Westland, S., "Colour-Imager Characterization by Parametric Fitting of Sensor Responses", Color Research and Applications, Vol. 26, Nr 6, Dec. 2001, (2001).

13. Natural Color System (NCS), Scandinavian Colour Institute, http://www.ncs.se

14. Romero, Javier, et. al, "Color-signal filtering in the Fourier-frequency domain", J. Opt. Soc. Am. A/Vol. 20, No. 9/September 2003, (2003).

15. Hardeberg, Jon Yngve, et. al, "Spectral characterization of electronic cameras", École Nationale Supérieure des Télécommunications, Paris, France.

16. Imai, Francisco H., Berns, Roy S., \& Tzeng, Di-Y, "A Comparative Analysis of Spectral Reflectance Estimated in Various Spaces Using a Trichromatic Camera System”, Journal of imaging and technology, Volume 44, Number 4, July/August 2000, (2000).

17. Imai, Francisco H., \& Berns, Roy S., "Spectral estimation of artist oil paints using multifilter trichromatic imaging", Proc. of the $9^{\text {th }}$ Congress of the International Colour Association, Rochester, NY, 2001, pp. 504-507, (2001). 